\title{
Transição nutricional na adolescência: uma abordagem dos últimos 10 anos
}

\author{
Nutritional transition in adolescence: an approach of the last 10 years
}

Transición nutricional en la adolescencia: un enfoque de los últimos 10 anos

\begin{abstract}
Diana Souza dos Santos ${ }^{1 *}$, Michele de Sousa Carneiro ${ }^{1}$, Sheila Cristina Martins e Silva ${ }^{2}$, Christian Nunes Aires ${ }^{2}$, Lorenn Juliet da Silva Carvalho ${ }^{1}$, Liliane Cristina Bandeira Costa1.
\end{abstract}

\section{RESUMO}

Objetivo: Avaliar a transição nutricional dos adolescentes nos últimos 10 anos. Métodos: A pesquisa foi realizada através da revisão bibliográfica, de artigos científicos, com base nos dados do Scielo, biblioteca virtual de saúde (BIREME), dados do SISVAN, MS e descritores em ciências da saúde (DeCs). Os artigos analisados consistem nos anos de 2004 a 2017. Resultados: A maioria dos artigos mostraram que houve a diminuição da desnutrição e aumento do excesso de peso (sobrepeso e obesidade), devido a vários fatores como: inserção da mulher no mercado de trabalho, diminuição da atividade física, mudanças nos hábitos alimentares, o avanço da tecnologia, dentre outros. Considerações finais: No presente estudo pode-se observar que a alimentação inadequada, acarretou o aparecimento de patologias relacionadas a má alimentação nesta faixa etária (adolescência). Verificou-se que essa grande proporção de adolescentes com excesso de peso, demonstra a necessidade urgente de medidas preventivas realizada pelas autoridades para formulação de diretrizes para políticas de saúde, para que esse quadro venha ser reduzido.

Palavras-Chaves: Consumo alimentar, Obesidade, Adolescente.

\section{ABSTRACT}

Objective: To evaluate the nutritional transition of adolescentes in the last 10 years. Methods: The research was fulfilled through of bibliographic review, scientific articles, based on data from Scielo, vitual health library (BIREME), SISVAN's data, MS and descriptors in health sciences ( DeCs). The articles analyzed consist of years from 2004 to 2017. Results: Most articles showed that there has been decrease in malnutrition and an increase in overweight ( overweight and obesity) due to factors, such as: Insertion of women in the labor Market, reduction of physical activity, changes in eating habits, the advancement of tecnology, among others. Finals considerations: In the present study it can be observed that the inadequate feeding resulted the appearance of pathologies related to poor diet in this age group (adolescence). It was verified that this large proportion of overweight teenagers, demonstrates the urgent necessity of preventive measures taken by authorities to formulate guidelines for health policies, so this framework will be reduced.

Keywords: Food consumption, Obesity, Adolescent.

\section{RESUMEN}

Objetivo: Evaluar la transición nutricional de los adolescentes en los últimos 10 años. Métodos: La investigación fue realizada a través de la revisión bibliográfica, de artículos científicos, con base en los datos del Scielo, biblioteca virtual de salud (BIREME), datos del SISVAN, MS y descriptores en ciencias de la salud (DeC). Los artículos analizados consisten en los años 2004 a 2017. Resultados: La mayoría de los artículos mostraron que la disminución de la desnutrición y el aumento del exceso de peso (sobrepeso y

\footnotetext{
${ }^{1}$ Faculdade Integrada Brasil Amazônia, FIBRA, Belém-PA. * E-mail: dianasantos72@hotmail.com
} 
obesidad), debido a varios factores como: inserción de la mujer en el mercado de trabajo, disminución de la actividad física, cambios en los hábitos alimentarios, el avance de la tecnología, entre otros. Consideraciones finales: En el presente estudio se puede observar que la alimentación inadecuada, acarreó la aparición de patologías relacionadas a mala alimentación en este grupo de edad (adolescencia). Se verificó que esta gran proporción de adolescentes con sobrepeso, demuestra la necesidad urgente de medidas preventivas realizadas por las autoridades para la formulación de directrices para políticas de salud, para que ese cuadro venga a ser reducido.

Palabras-Claves: Consumo Alimentario, Obesidad, Adolescente.

\section{INTRODUÇÃO}

A população brasileira, nas últimas décadas, vem passando por grandes transformações sociais que resultaram em mudanças no seu padrão de saúde e consumo alimentar. Essas transformações acarretaram impacto na diminuição da pobreza e exclusão social com consequente redução da fome e escassez de alimentos, com melhoria ao acesso e variedade dos mesmos, além da garantia da disponibilidade média de calorias para o consumo, embora ainda existam cerca de 16 milhões de brasileiros vivendo na pobreza extrema. A diminuição da fome e da desnutrição veio acompanhada do aumento crescente da obesidade em todas as camadas da população, apontando para um novo cenário de problemas relacionados à alimentação e nutrição (BRASIL, 2013).

Vários fatores contribuíram para ocorrência da transição nutricional nas últimas décadas, como a inserção da mulher no mercado de trabalho, o crescimento tecnológico da indústria alimentícia na produção de alimentos ultra processados, que embora sejam práticos, são prejudiciais à saúde, porque contribuem para o aumento do consumo de gorduras, açúcares, sal, corantes, conservantes e muitas outras substâncias que promovem o desenvolvimento da obesidade. Todos estes fatores auxiliaram as mudanças nos hábitos alimentares aliados a falta da atividade física, entre outros (SOUZA, 2010).

Porém, no Brasil ainda são escassas as pesquisas relacionadas ao estado nutricional e a transição nutricional da população, onde antes predominava a desnutrição e nas últimas décadas vem aumentando o excesso de peso - sobrepeso e obesidade. Em outras palavras, o País tem vivenciado uma peculiar e rápida transição nutricional, onde antes apresentava altas taxas de desnutrição, na década de 1970, e passou a ser um país com metade da população adulta com excesso de peso, em 2008, e é considerado atualmente um dos maiores problemas de saúde pública, afetando todas as faixas etárias de idade (BRASIL, 2017).

Devido à escassez de artigos sobre o tema em questão, a presente pesquisa se propõe a realizar uma revisão bibliográfica, com o objetivo de verificar a ocorrência da transição nutricional em adolescentes no Brasil nos últimos anos.

\section{METODOLOGIA}

A presente pesquisa foi realizada em agosto de 2018 através da revisão bibliográfica, de artigos científicos, a busca nos bancos de dados foi realizada utilizando às terminologias cadastradas nos DeCs, BIREME, Scielo, SISVAN e MS. As palavras-chaves utilizadas na busca foram consumo alimentar, obesidade e adolescente.

Os artigos para a pesquisa foram selecionados entre os anos de 2004 a 2018, dentre os 30 artigos selecionados, analisou-se 18 artigos pois os demais não abordavam o tema da forma como proposto pela pesquisa. 


\section{RESULTADOS E DISCUSSÃO}

Foram selecionados 30 artigos dentre os quais foram analisados apenas 18 , pois foi verificado que os demais não abordavam o tema da forma como proposto pela pesquisa, as fontes utilizadas foram dos bancos de dados do DeCs, BIREME, Scielo, SISVAN e MS.

Dentre os artigos analisados observamos que desde 2004 a 2015, ocorreu uma diminuição da desnutrição e aumento do sobrepeso e obesidade (LEVI et al., 2010). Essa mudança se chama transição nutricional, que consiste em um processo de modificações sequenciais no padrão de nutrição e consumo, que acompanha mudanças econômicas, sociais, demográficas e mudanças do perfil de saúde das populações (PINHEIRO, FREITAS, CORSO, 2004).

A adolescência é uma fase de grandes conturbações que podem ser originadas por hábitos familiares, amizades, valores e regras sociais e culturais, essas conturbações podem repercutir sobre 0 comportamento alimentar, autoimagem, saúde individual e desenvolvimento psicossocial. Os estudos analisados identificaram hábitos alimentares inadequados, que normalmente vieram da infância; e se torna fatores de risco para o desenvolvimento de doenças na fase da adolescência (LEVI et al., 2010)

A desnutrição consiste quando uma pessoa recebe uma alimentação insuficiente quantitativamente para suprir suas necessidades fisiológicas, ou seja, falta de nutrientes como minerais vitaminas e macro nutrientes, e o nosso órgão controlador de toda a atividade metabólica, que é o sistema nervoso, se "programa" permanentemente para economizar energia em forma de gordura e reduzir o crescimento, para garantir a sobrevivência em condições adversas (SAWAYA, 2010).

O excesso de peso é caracterizado quando o Índice de Massa Corporal (IMC) é maior ou igual a $25 \mathrm{~kg} / \mathrm{m} 2$, e está associado a outras desordens manifestadas pelo excesso de gordura corporal. As condições mais frequentemente associadas ao excesso de peso são as dificuldades respiratórias, problemas dermatológicos, distúrbios do aparelho locomotor, dislipidemias, doenças cardiovasculares, diabetes, outros fatores que contribuir para essa condição são: sedentarismo, falta de atividade física, má alimentação dentre outros (VELOSO, SILVA, 2010).

O quadro abaixo apresenta o perfil nutricional de adolescentes no Brasil no ano de 2016 (Quadro 1).

Quadro 1 -Perfil nutricional de adolescentes do Brasil no ano de 2016.

\begin{tabular}{|c|c|c|}
\hline IMC X IDADE & Quantidade (Pessoas) & $\%$ \\
\hline Magreza Acentuada & 33.595 & 1.07 \\
\hline Magreza & 93.867 & 3 \\
\hline Eutrofia & 2.232 .824 & 71.31 \\
\hline Sobrepeso & 539.056 & 17.22 \\
\hline Obesidade & 192.950 & 6.16 \\
\hline Obesidade Grave & 38.720 & 1.24 \\
\hline Total & $\mathbf{3 . 1 3 1 . 0 1 2}$ & $\mathbf{1 0 0}$ \\
\hline
\end{tabular}

Fonte: Adaptada do SISVAN, 2016.

A obesidade é considerada uma síndrome metabólica, que consiste no acúmulo excessivo de gordura corporal, que acarreta prejuízos a saúde dos indivíduos. Essa síndrome é considerada um grande problema de saúde pública em países desenvolvidos e uma epidemia global pela organização mundial de saúde (OMS), adolescente com obesidade geralmente sofre com baixa autoestima, compromete o desempenho escolar e relacionamentos com amigos e familiares que podem desencadear consequências psicológicas (CARVALHO et al. 2012). 
Os autores relatam que a carga de morbimortalidade dos países subdesenvolvidos é maior do que nos países em desenvolvimento, também foi observado que as doenças crônicas não transmissíveis aumentam e fatores que podem ter desencadeado esse aumento são: uma alimentação hipercalórica, aumento do acesso ao consumo de alimentos, o processo de urbanização, o sedentarismo e a inserção da mulher no mercado de trabalho, levando ao maior consumo de alimentos industrializados e congelados, avanços das tecnologias e à diminuição acentuada no consumo de alimentos mais saudáveis (verduras, legumes, e frutas) (FILHO et al. 2008).

A inserção da mulher no mercado de trabalho ocasionou uma família economicamente dependente da participação da renda da mulher no sustento da família, com isso perde-se um pouco a figura da mulher dona do lar, o que transformar a qualidade da alimentação uma vez que a mesma não dispõe de tanto tempo para preparar as refeições com tanta qualidade, preferindo alimentos industrializados, e até mesmo a realização das refeições fora do lar, principalmente em restaurantes, pensões e fast-foods (SOUZA, 2010). Como pode ser observado no quadro 2 a seguir.

Quadro 2 - Alimentos ultraprocessados.

\begin{tabular}{|l|c|}
\hline \multicolumn{1}{|c|}{ Alimentos } & \% em calorias \\
\hline Bolos, tortas e biscoitos doces & 3,0 \\
\hline Lanche do tipo fast foods & 2,9 \\
\hline Refrigerante refrescos & 2,6 \\
\hline Pães de forma, de hambúrguer e de hot dog & 2,4 \\
\hline Guloseimas & 2,2 \\
\hline
\end{tabular}

Fonte: Adaptado de Louzada et al, 2015.

Concomitante com a alteração da característica da dieta observou-se uma redução do nível de atividade física, vários fatores podem explicar esse fato mais as principais mudanças estão nas distribuições das ocupações setoriais (da agricultura para as indústrias) e um aumento do número de trabalhos com redução do esforço ocupacional, em virtude do aumento da tecnologia (SOUZA, 2010).

Observamos também a diminuição das atividades de lazer como práticas esportivas e longas caminhadas, para atividades sedentárias, como televisão, vídeo game ou computador. A utilização de recursos tecnológicos também contribui efetivamente para o aumento do sedentarismo da população, portões eletrônicos, escadas rolantes, vidros automáticos, veículos motorizados, (SOUZA, 2010).

Em resposta as modificações no padrão de comportamento alimentar e da drástica redução na pratica de atividade física, resultou-se no aumento acelerado de adolescentes com, sobre peso e obesidade. Sendo assim, o combate efetivo deste problema de saúde pública requer estratégias de curto prazo no que diz respeito à proteção, promoção e apoio a estilos de vida saudáveis (SOUZA, 2010).

De acordo com o SISVAN (2016) $17,22 \%$ dos adolescentes apresentavam sobrepeso e $6,16 \%$ estava obeso, o que equivale a $23,38 \%$ do total de indivíduos avaliados. Valores superiores ao encontrado por Leal et al., (2012), onde a prevalência de excesso ponderal foi de 13,3\%, sendo $9,5 \%$ de sobrepeso e 3,8\% de obesidade. E diverge muito dos resultados de Flores et al., (2013), no qual o sobrepeso estava presente em $24,1 \%$ dos adolescentes do sexo masculino e $22 \%$ no sexo feminino e a obesidade está em $5,5 \%$ do sexo masculino e $6 \%$ do sexo feminino. Porém, convém ao encontrado por Ramires et al., (2014), onde a classificação de sobrepeso e obesidade corresponde a $24 \%$ da amostra estudada.

Em relação ao consumo de produtos alimentícios ultraprocessados, Louzada et al. (2015) afirmam que bolos, tortas e biscoitos doces ocupam $3 \%$ das calorias totais dos adolescentes estudados. No entanto, 0 estudo Teo et al (2014), indica que 69,9\% dos adolescentes consomem doces mais que duas vezes por 
semana, o que é considerado pelo mesmo um consumo regular. No estudo de Santana (2016) 45, 9\% dos adolescentes referem consumo de ricos em açúcar 5 vezes ou mais por semana, o que também é considerado um consumo regular.

O consumo de refrigerante no estudo de Louzada et al. (2015) representa 2,6\% das calorias totais dos adolescentes, porém Marques et al. (2016) avaliaram a alimentação de 645 adolescentes e 53,5\% tinha o consumo mais que duas vezes por semana. No estudo de Santana (2016) $42,8 \%$ dos avaliados declararam consumo de 5 vezes ou mais por semana.

Louzada afirma que a ingestão de pão representa $2,4 \%$ das calorias totais dos adolescentes, o que é visto como relativamente baixo. Concordando com Messias et al. (2016), onde apenas 12, 57\% do sexo feminino e 14, 20\% do sexo masculino afirmam ter o consumo de pão. Discordando de Martins et al. (2014) onde quase metade dos avaliados $(40,1 \%)$ afirmam a ingestão deste tipo de alimento

\section{CONSIDERAÇÕES FINAIS}

Neste estudo foi possível observar que a alimentação inadequada acarreta uma serie de complicações, levando a diminuição progressiva da desnutrição para o aumento do excesso do peso, sobrepeso e obesidade. A partir das pesquisas verificou-se que essa grande proporção de adolescentes com excesso de peso, demonstra a necessidade urgente de medidas preventivas realizadas pelas autoridades para formulação de diretrizes para políticas de saúde, para que esse quadro venha ser reduzido. Dentre as estratégias para a melhoria da saúde pública podem se destacar: ações de educação alimentar, conduzida pelo nutricionista, e incentivo à prática de atividades físicas diárias.

\section{REFERÊNCIAS}

1. BRASIL. Ministério da Saúde. Sistema de Vigilância Alimentar e Nutricional - SISVAN: Relatório do Estado Nutricional de adolescentes no Brasil / Ministério da Saúde, Sistema de Vigilância Alimentar e Nutricional - SISVAN 2016. Disponível em: dabsistemas.saude.gov.br/sistemas/sisvan. Acesso em: 09 de março de 2017.

2. BRASIL. Ministério da Saúde. Secretaria de Atenção à Saúde. Departamento de Atenção Básica. Portal do Departamento de Atenção Básica. Prevenção e Controle de Agravos Nutricionais, 2017. Disponível em: http://dab.saude.gov.br/portaldab/ape_pcan.php. Acesso em: 09 de maio de 2017.

3. BRASIL. Ministério da Saúde. Secretaria de Atenção à Saúde. Departamento de Atenção Básica. Política Nacional de Alimentação e Nutrição / Ministério da Saúde, Secretaria de Atenção à Saúde. Departamento de Atenção Básica. Básica. - 1. ed., Brasília: Ministério da Saúde, 2013. 84 p.

4. CARVALHO E, SIMÃO M, FONSECA M et al. Obesidade: aspecto epidemiológico e prevenção. Revista de Medicina de Minas Gerais. 2013, Minas Gerais, v.23, n.1, p.74-82.

5. FILHO M, SOUZA A, MIGLIOLI T et al. Anemia e obesidade: um paradoxo da transição nutricional brasileira. Caderno de Saúde Pública. 2008, Rio de Janeiro, v.24, n.2, p.247-257.

6. FLORES L, GAYA A, PETERSEN R et al. Tendência do baixo peso, sobrepeso e obesidade de crianças e adolescentes brasileiros. Jornal de Pediatria. 2013, Rio de Janeiro, 89:456-61.

7. LEAL V, LIRA P, OLIVEIRA J et al. Excesso de peso em crianças e adolescentes no Estado de Pernanbuco, Brasil: prevalências e determinantes. Cadernos de Saúde Pública, 2012, Rio de Janeiro, v.28, n.6, p.1175-1182

8. LEVY R, CASTRO I, CARDOSO L et a. Consumo e comportamento alimentar entre adolescentes brasileiros: pesquisa nacional da saúde da escola (PNSE), 2009. Ciência \& saúde coletiva, 2010, São Paulo, v.15, n.2, p.3085-3097.

9. LOUZADA M, MARTINS A, CANELLA D et al. Alimentos ultraprocessados e perfil nutricional da dieta no Brasil. Revista de Saúde Pública, 2015, São Paulo, v.49, n.38, p.01-11.

10. MARQUES A, BRAGA I, OLIVEIRA E et al. Consumo de alimentos ricos em sódio e alteração dos níveis pressóricos em adolescentes de Itaqui/RS. Revista da AMRIGS, 2016, Porto Alegre, v. 60, n.2, p.97-103.

11. MARTINS C, SOUZA S, REIS I et al. Consumo de alimentos entre adolescentes de um estado do Nordeste brasileiro. DEMETRA: Alimentação, Nutrição \& Saúde, 2014, v.9, n.2, p.577-594, outubro. Disponível em: http://www.epublicacoes.eurj.br/ojs/index.php/demetra/article/view/9693.

12. MESSIAS C, SOUZA H, REIS I. Consumo de alimentos ultraprocessados e corantes alimentares por adolescentes de uma escola pública. Adolescência \& Saúde, Rio de Janeiro, 2016 v.13, n.4, p. 7-14.

13. PINHEIRO A, FREITAS S, CORSO A. Uma abordagem epidemiológica da obesidade. Revista de Nutrição, 2004, Florianópolis, v.17, n.4, p.523-533.

14. RAMIRES E, MENEZES R, OLIVEIRA $\mathrm{J}$ et al. Estado nutricional de crianças e adolescentes de um município do semiárido do Nordeste brasileiro. Revista Paulista de Pediatria, 2014, São Paulo, v.32, n.3. p.200-207.

15. SANTANA SO. Caracterização do consumo alimentar e seus determinantes entre adolescentes de escolas públicas e privadas das capitais brasileiras e do Distrito Federal. Dissertação (Mestrado em Nutrição Humana) - Universidade de Brasília, Brasília, $2014 ; 96$ p.

16. SAWAYA AL. Consequências em longo prazo e efeitos da recuperação nutricional. Estudos Avançados, São Paulo, 2006, v.20, n.58, p.147-158.

17. SOUZA, Elton Bicalho de. Transição nutricional no Brasil: analise dos principais fatores. Caderno UNIFOA, Volta Redonda, Ano V, n. 13, agosto 2010. Disponível em: http://www.unifoa.edu.br/caderno/edicao/13/49.pdf

18. VELOSO H, SILVA A. Prevalência e fatores associados à obesidade e ao excesso de peso em adultos maranhenses. Revista Brasileira de Epidemiologia, São Paulo, 2010, v.13, n.3, p.400-412. 